\title{
Subjective adaptive correction of the aberrations of the human eye
}

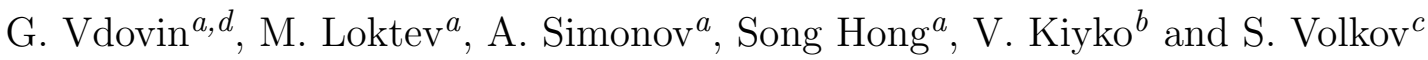 \\ ${ }^{a}$ TU Delft, Mekelweg 4, 2628 CD, Delft, The Netherlands \\ ${ }^{b}$ General Physics Institute, Vavilov str. 38, 117942, Moscow, Russia \\ ${ }^{c}$ St-Petersburg IFMO, Sablinskaya str. 14, St Petersburg 197101, Russia \\ ${ }^{d}$ OKO Technologies, PO Box 581, 2600 AN Delft, The Netherlands
}

\begin{abstract}
We designed and built an experimental setup for subjective (manually controlled) correction of up to 12 orthogonal terms (excluding tilts and defocus) of the aberration of the human eye. In our experiments, the subject was looking through an adaptive optical system at the USAF resolution chart. By using the arrow keys of the computer keyboard, the subject was able to control the amplitudes of up to 12 orthogonal aberration terms, introduced by a deformable mirror, optically conjugated to the pupil of the eye. Preliminary statistical analysis on a group of 12 participants with 6 correction attempts per participant, demonstrated satisfactory correlation of aberration coefficients obtained by the same person in different correction attempts. The majority of the participants were able to improve the visual acuity by subjective optimization of the figure of the deformable mirror.
\end{abstract}

Keywords: Adaptive optics, deformable mirror, wavefront sensing, ophthalmic optics, human eye

\section{INTRODUCTION}

Recently it has been shown that it is possible to measure the aberrations of the human eye ${ }^{1}$ and to improve the resolution and visual acuity by correcting the aberrations measured..$^{2-4}$

Traditional correction methods, such as spectacles, are usable only for defocus and astigmatism. Corrections of higher-order aberrations are sensitive to the misalignment between the optical axes of the eye and the correction optic. ${ }^{5,6}$ To correct the higher-order aberrations, the corrector must be optically conjugated to the eye, regardless of the eye movements. Technically such a corrector can be implemented as an implantable multichannel liquid crystal lens with several degrees of freedom. ${ }^{7}$ To control different correction modes, such a device will require multichannel feedback.

To implement such a multichannel control, a trial-and-error approach can be used that consists of subjectively controlled correction of different low-and-high order aberrations, aimed at subjective improvement of the visual acuity. For such a method, it is important to have a system that is able to introduce orthogonal aberration modes, as this will greatly improve the convergency of the subjective optimization process.

\section{GENERATION OF ZERNIKE MODES}

\subsection{Deformable mirror}

To correct the aberrations we used a $19 \mathrm{ch} 30 \mathrm{~mm}$ piezoelectric deformable mirror supplied by OKO Technologies. The mirror consists of 19 piezoelectric tube actuators mounted on the base holder. Reflective plate is bonded to the top of the actuator structure and coated to form the mirror. The mirror shape is controlled by a high-voltage amplifier board. The board contains 20 non-inverting DC amplifiers with gain 59. The control voltages are generated by 24-ch 8-bit PCI boards. The output voltage for each amplifier can take any of 255 discrete values, in steps of $\sim 1.25 \mathrm{~V}$. This discrete control produces $30 \mathrm{~nm}$ steps with free unloaded actuators, the displacements

Further author information: (Send correspondence to Gleb Vdovin)

E-mail: gleb@okotech.com, Telephone: +31-62-2794647 


\begin{tabular}{|l|l|}
\hline \hline Parameter & Value \\
\hline \hline Aperture shape & circular $30 \mathrm{~mm}$ in diameter \\
\hline Mirror coating & $\mathrm{Al}$ \\
\hline Actuator voltages & $-50+350 \mathrm{~V}$ (with respect to the ground electrode) \\
\hline Number of actuators & $19 \mathrm{in}$ a hexagonal arrangement \\
\hline Actuator capacitance $C_{a}$ & $13 \mathrm{nF}$ \\
\hline Initial RMS deviation from reference sphere & less than $0.8 \mu \mathrm{m}$ \\
\hline Main initial aberration & astigmatism \\
\hline Frequency range & $2 \mathrm{kHz}(4 \mathrm{kHz}$ first resonance) \\
\hline Maximum stroke & $9 \mu \mathrm{m}$ \\
\hline Actuator pitch & $6 \mathrm{~mm}$ \\
\hline & better than $9 \%$ \\
\hline Hysteresis &
\end{tabular}

Table 1. Parameters of the piezoelectric mirror used in the experiment.

of the mirror surface are usually much smaller due to the stiffness of the faceplate and rigidity of the neighbor actuators. Since the minimum control step is much smaller than $\lambda / 12$ at any visible wavelength, the precision of the 8-bit control is more than sufficient.

The technical parameters of the mirror are shown in the table 2.1.

The photograph of the mirror is shown in Fig. 1.

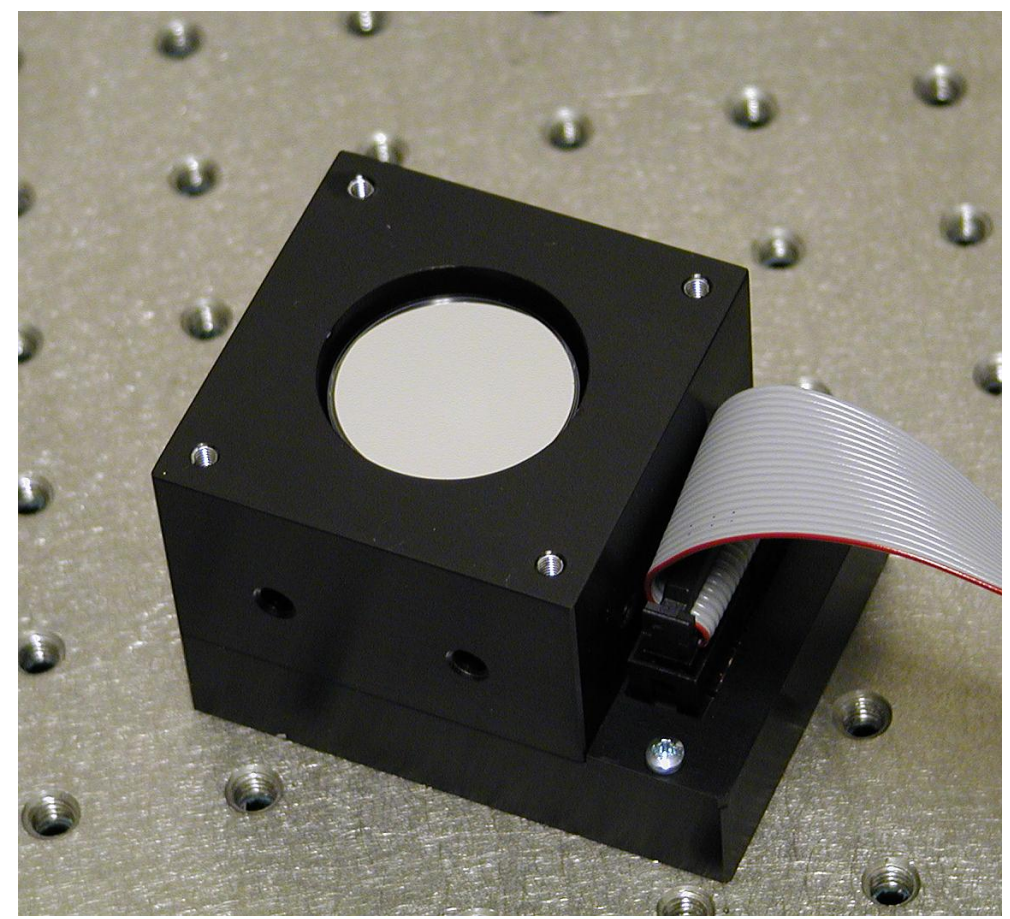

Figure 1. 19-ch piezoelectric deformable mirror.

\subsection{Microlens array}

In our experiments we used OKO-127 microlens array with hexagonal arrangement of 127 microlenses in a $3.5 \mathrm{~mm}$ light aperture. The array geometry was precise enough to use it as a reference, without calibrating with reference 

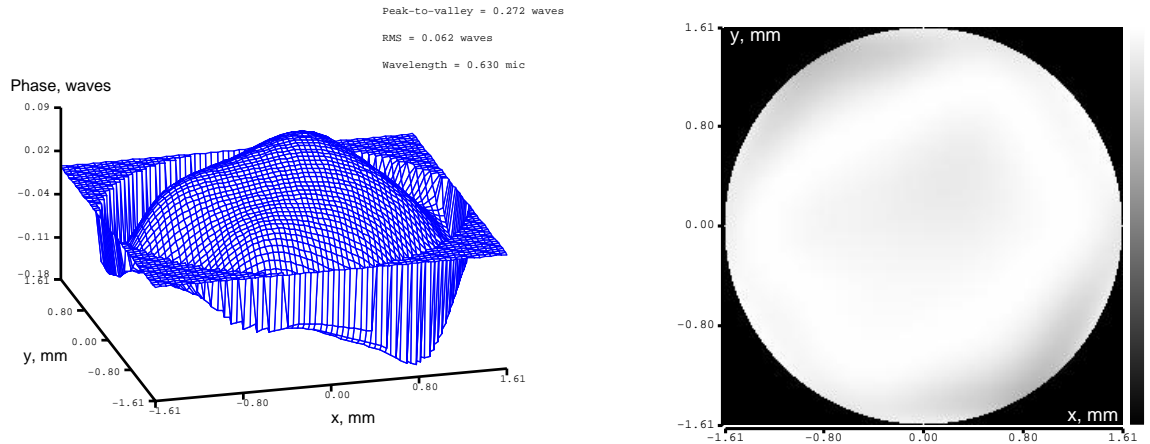

Figure 2. Wavefront sensor error due to the irregularity of the microlens array; wavelength $0.63 \mu \mathrm{m}$.

wavefront. The measured wavefront error of the sensor, referenced to the geometry of the microlens array, is shown in Figure 2; this error (not exceeding $\lambda / 12$ ) is due to the lens pitch irregularity. As the precision is good enough for ophthalmic experiments, we did not use any separate reference pattern to calibrate the sensor in our experiments, although the software used in the experiment allows for measurements, referenced to any arbitrary spot pattern.

\subsection{Deformable mirror in the feedback loop}

Interferometric patterns of the mirror, with zero control applied, are shown in Fig. 3.
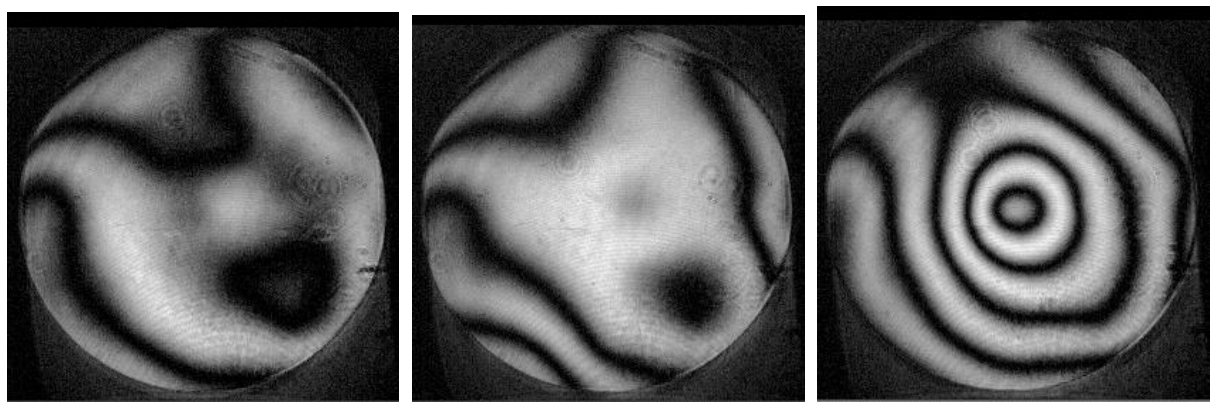

Figure 3. The initial optical figure of the deformable mirror with all actuators off (left); same with bias voltage applied to all actuators (center); same with a voltage applied to the central actuator and zero voltage allied to other actuators (right).

Experimental singular values for the piezoelectric deformable mirror are given in Figure 4; the first 15 orthonormal modes are shown in Figure 5.

The FrontSurfer wavefront control and reconstruction software ${ }^{8}$ performs the wavefront correction in a series of iterations based on the data read in real time from a Hartmann-Shack wavefront sensor. If the residual aberration $\phi_{n}$ at the $n$-th iteration corresponds to the set of actuator signals $\mathbf{X}_{n}$ then the actuator signals at the next step $\mathbf{X}_{n+1}$ will be determined by expression

$\mathbf{X}_{n+1}=\mathbf{X}_{n}-g \mathbf{A}^{-1} \phi_{n}$,

where $g$ is the feedback coefficient with value in the range (0..1], $\mathbf{A}$ is the influence matrix of the mirror, $\mathbf{A}^{-1}$ is its pseudo-inverse given by

$\mathbf{A}^{-1}=\mathbf{V S}^{-1} \mathbf{U}^{T}$

$\mathbf{U}, \mathbf{S}$ and $\mathbf{V}$ are the singular value decomposition (SVD) of $\mathbf{A}$ which is $\mathbf{A}=\mathbf{U S V}^{T} .{ }^{9}$ The columns of the matrix $\mathbf{U}$ make up an ortho-normal set of the mirror deformations (modes), and the values of the diagonal 


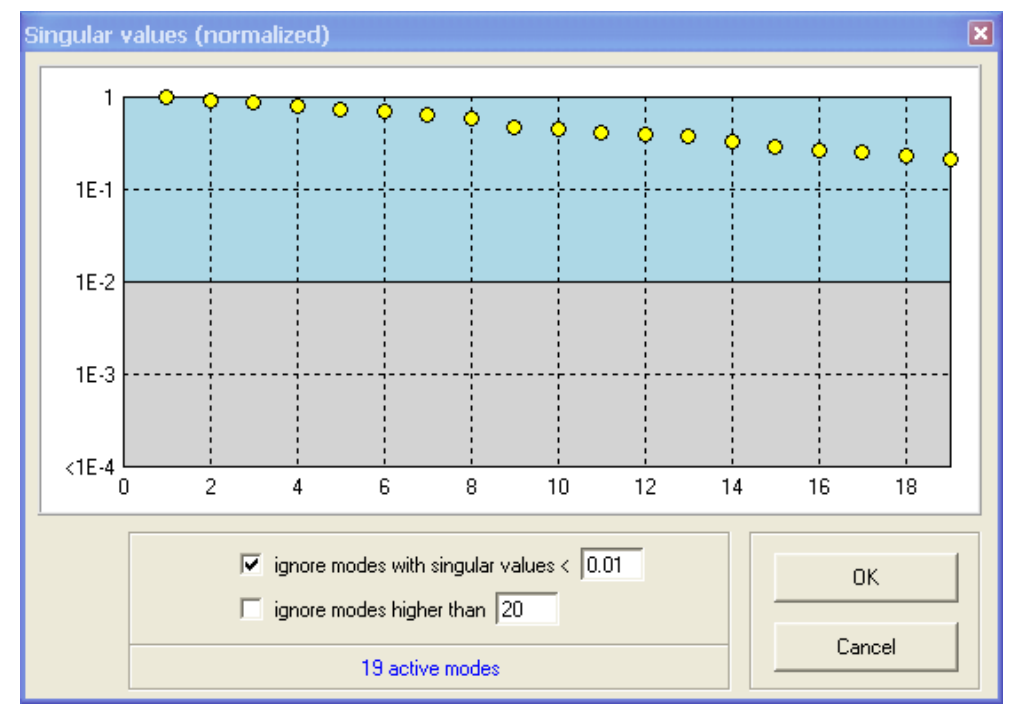

Figure 4. Measured singular values of 19-channel piezoelectric mirror.
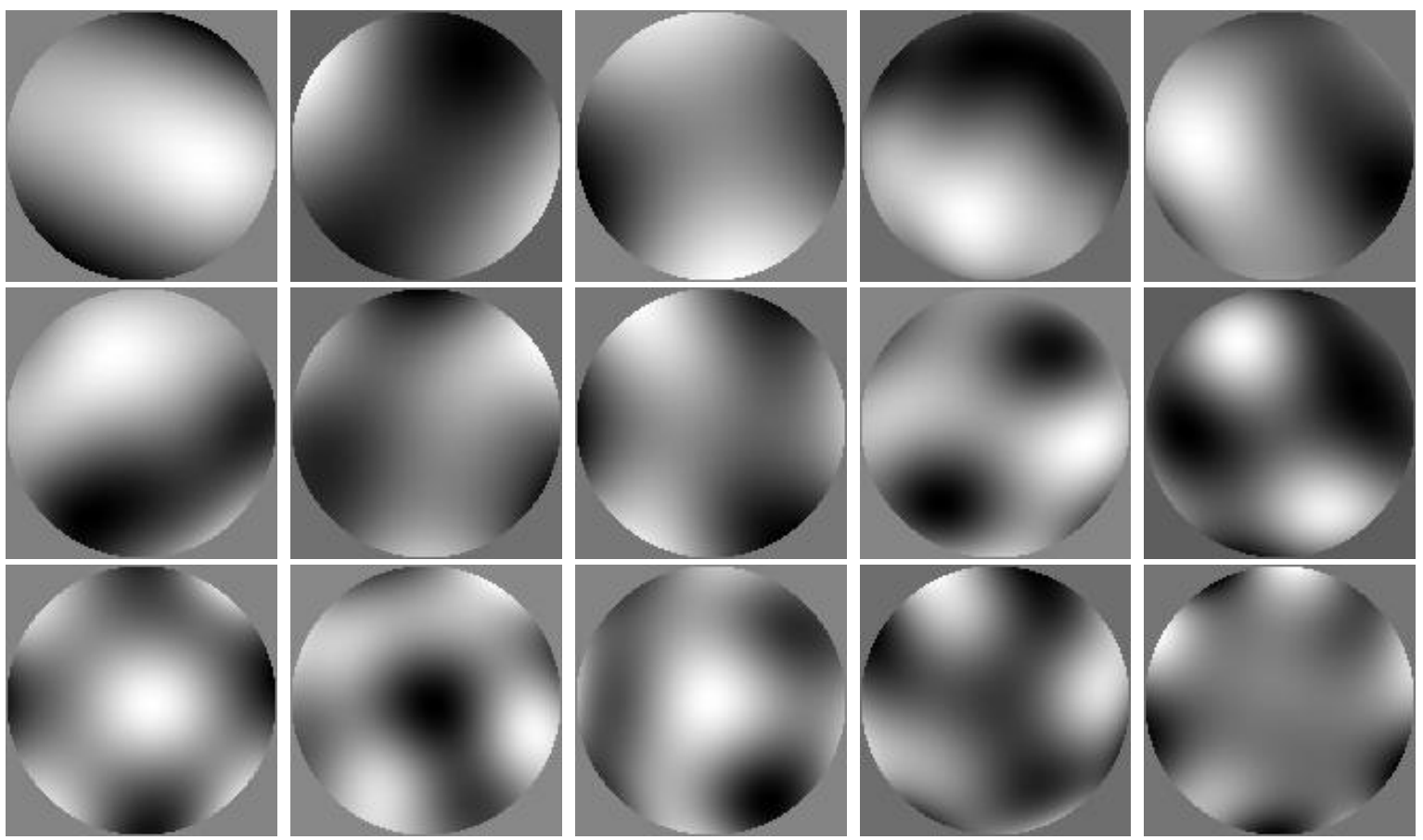

Figure 5. First 15 modes of a 19-channel piezoelectric mirror, obtained as a result of SVD-based orthogonalization. 

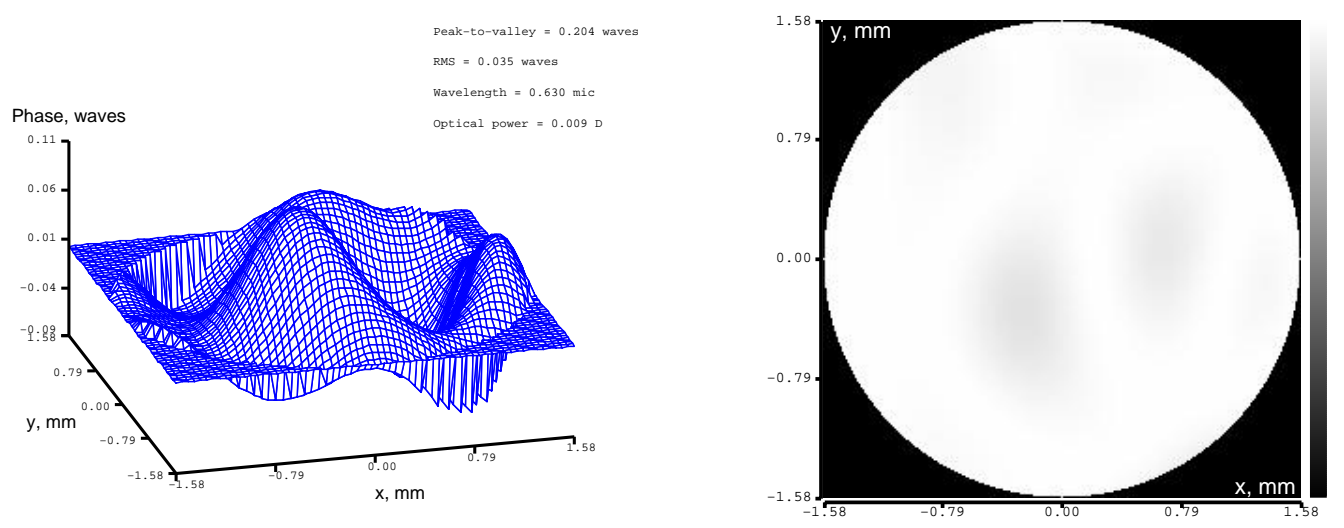

Figure 6. Optimization with respect to the reference mirror; residual aberrations.
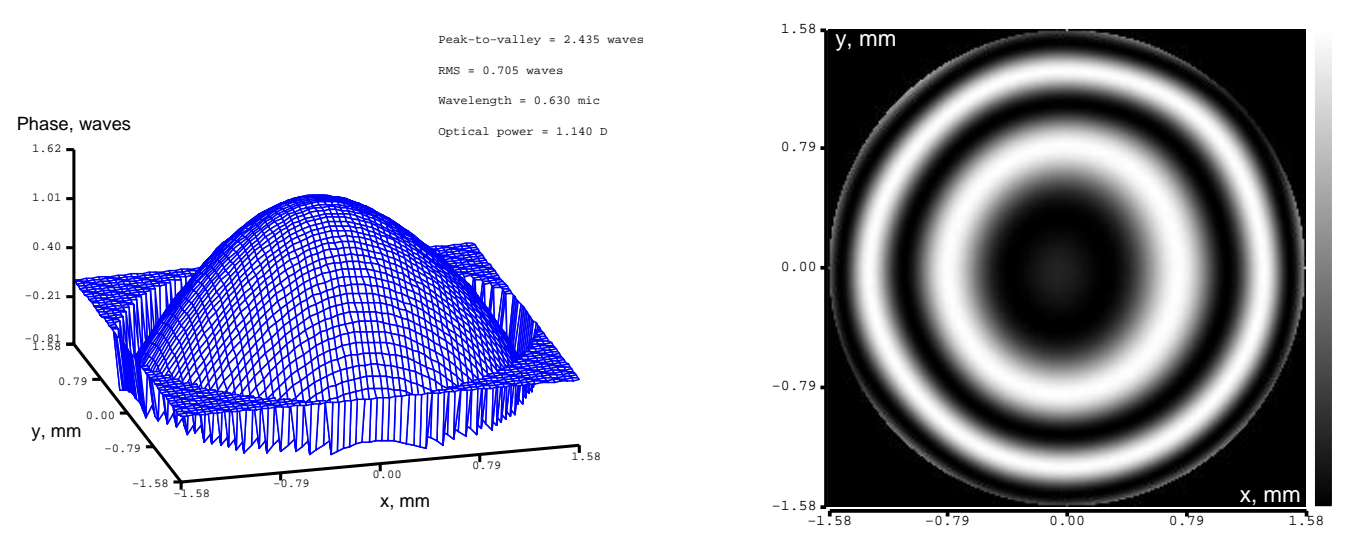

Figure 7. Generated defocus, Zernike term Z[2,0], amplitude $1 \mu \mathrm{m}$.
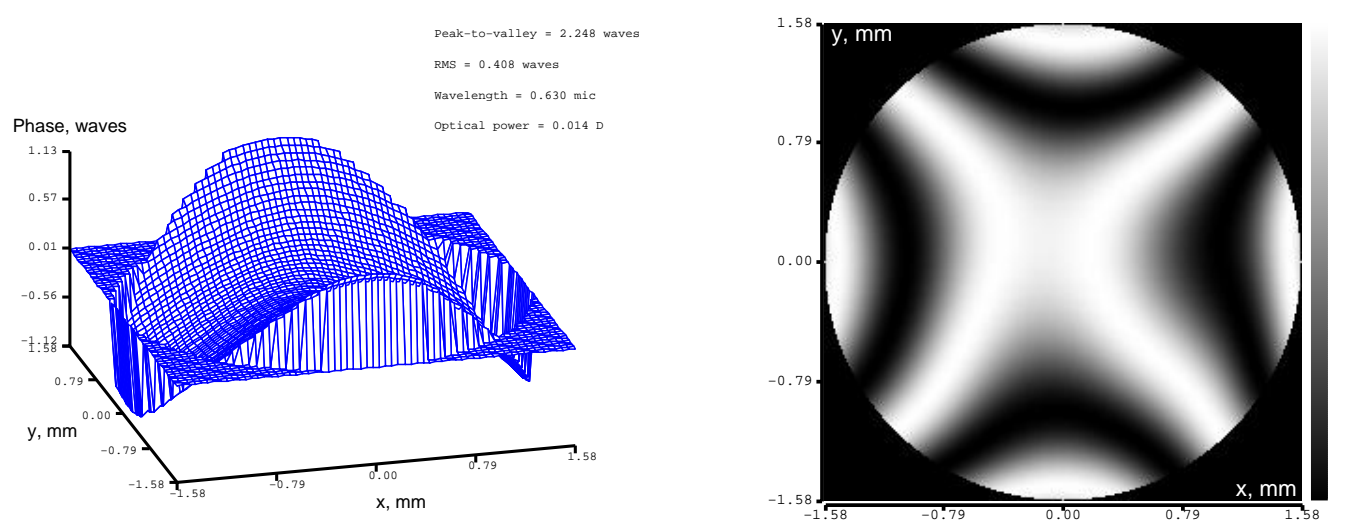

Figure 8. Generated astigmatism, Zernike term Z[2,2], amplitude $1 \mu \mathrm{m}$. 

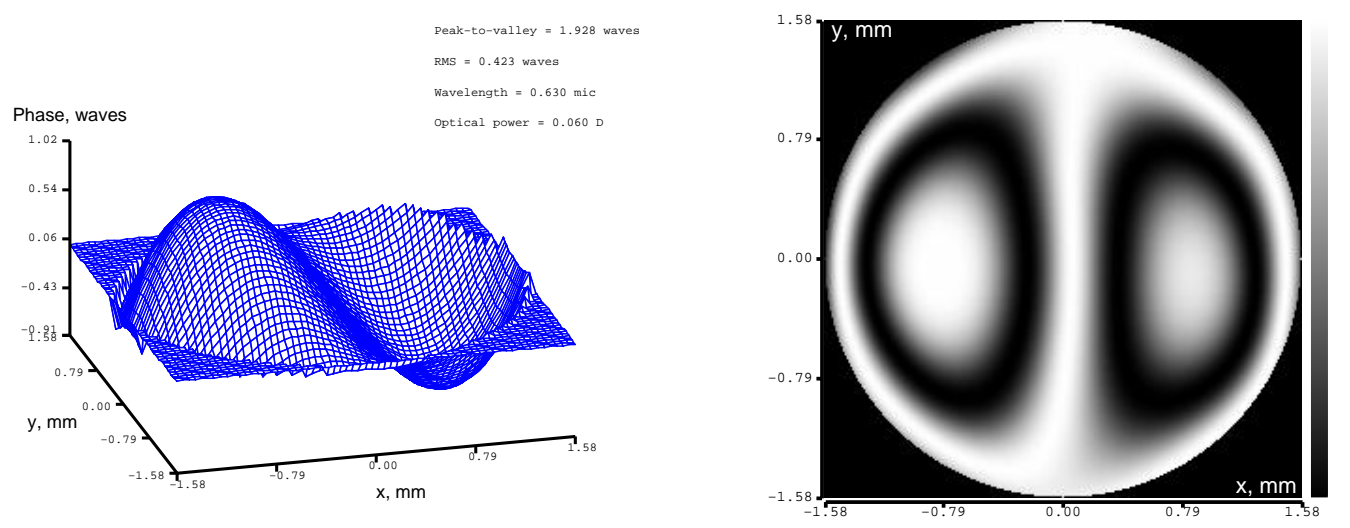

Figure 9. Generated coma aberration, Zernike term Z[3,1], amplitude $1 \mu \mathrm{m}$.
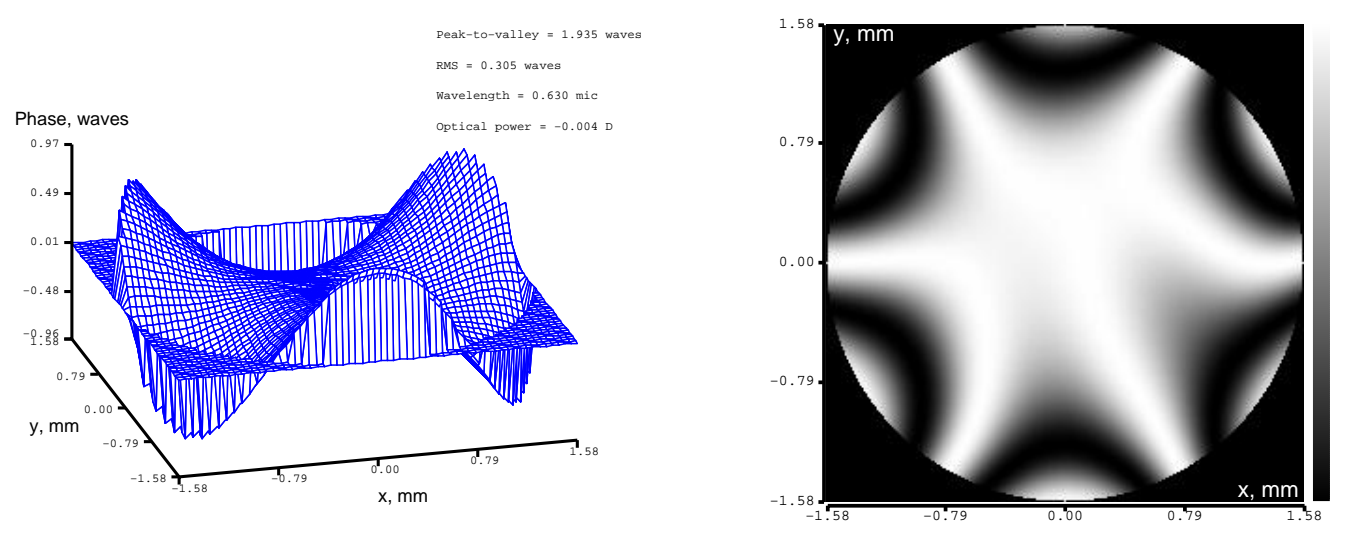

Figure 10. Generated trifoil aberration, Zernike term Z[3,3], amplitude $1 \mu \mathrm{m}$.
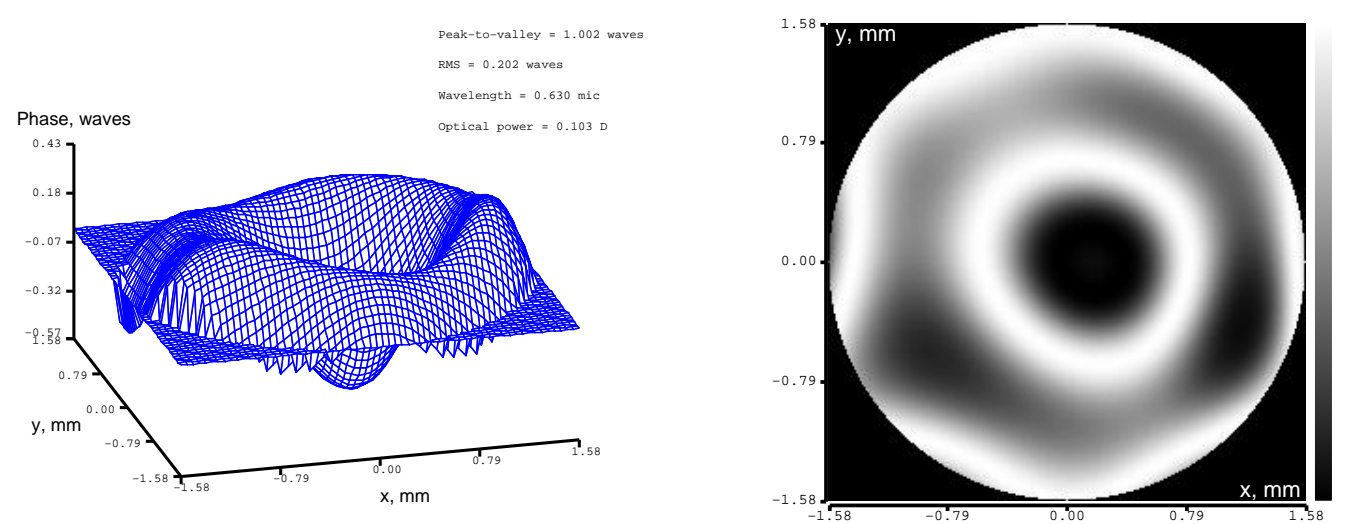

Figure 11. Generated spherical aberration, Zernike term Z[4,0], amplitude $0.5 \mu \mathrm{m}$. 

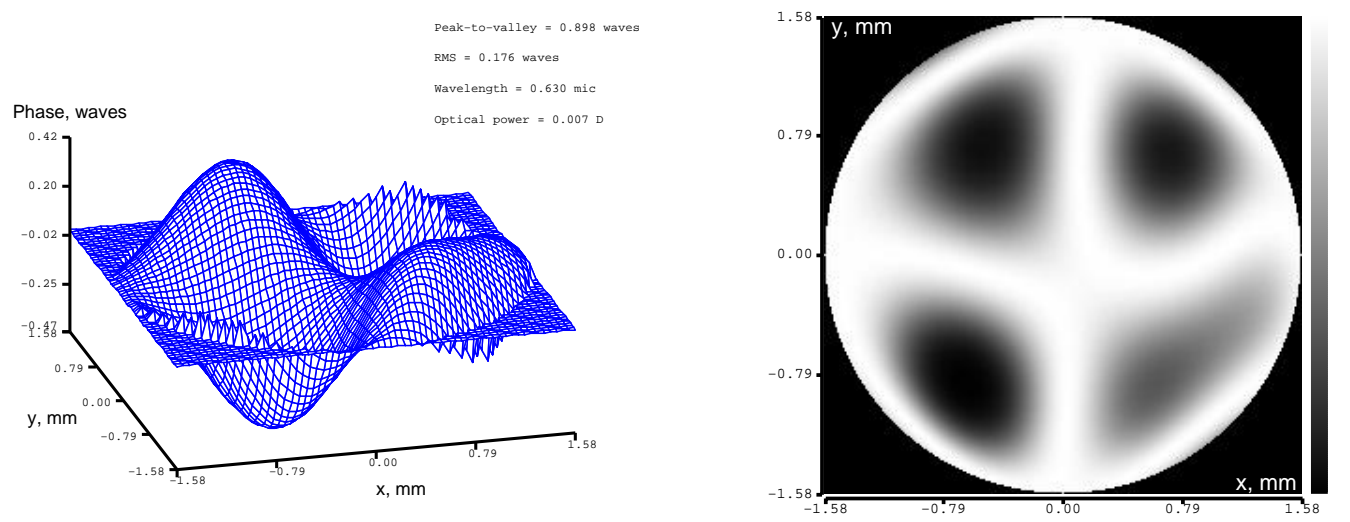

Figure 12. Generated Zernike term Z[4,2], amplitude $0.5 \mu \mathrm{m}$.

matrix $\mathbf{S}$ represent the gains of these modes. Discarding the modes with small singular values usually improves the controllability of the system but reduces the spatial bandwidth of the aberrations corrected.

The feedback is usually started from the initial shape of the mirror, obtained with setting all actuators to zero - see Fig. 3. During the feedback operation, the shape of the deformable mirror was flattened, the residual aberrations are shown in Figure 6. In the following tests we generated various Zernike aberrations; the results are presented in Figures 7-12.

The feedback system described above allows generation of arbitrary combination of low-order Zernike modes, with good precision and very high repeatability. The shape of the mirror is controlled by a feedback with frame frequency of the order of $\sim 30$ frames per second and allows of direct manual adjustment to any combination of Zernike terms. These arbitrary combinations of Zernike polynomials were used in our experiments on subjective acuity correction, described in the next section.

\section{SUBJECTIVE ACUITY CORRECTION}

To investigate the effectiveness of subjective correction of visual acuity, using a set of low-order Zernike polynomials, we set up the breadboard shown in Fig. 13. The setup consists of two feedback loops:

- A subjective feedback loop, consisting of a deformable mirror conjugated to the pupil of the human eye, and a test object USAF1951 placed at the infinity of a telescope. The human participants can correct the aberrations of his/her eye by changing the amplitude of the orthogonal modes of the deformable mirror. The control was realized through the computer keyboard; the "UP" key increased the amplitude of a certain mode, the "DOWN" key decreased the amplitude, and the "LEFT" and "RIGHT" keys were used to switch between the aberration modes.

- The second feedback loop consists of a deformable mirror - common with the subjective loop - and the Hartmann-Shack sensor positioned in the conjugated plane. This feedback system runs at a frame rate of $\sim 30 \mathrm{~Hz}$ and compensates the nonlinearity, hysteresis and creep of the deformable mirror. The system is also used to generate orthogonal modes on the mirror surface.

After the subjective correction cycle, we used the Shack-Hartmann sensor with 127 microlenses to objectively measure the figure of the adaptive mirror corresponding to the best correction achieved in the process of subjective manual feedback.

In our experiments we used both Zernike polynomials and system modes obtained as a result of singular value decomposition in the feedback loop with a Hartmann sensor. The correction results were estimated in terms of subjective vision improvement; at the same time, the optical figure of the deformable corrector was measured objectively. We assume that the method has a practical value if it satisfies the following criteria: 


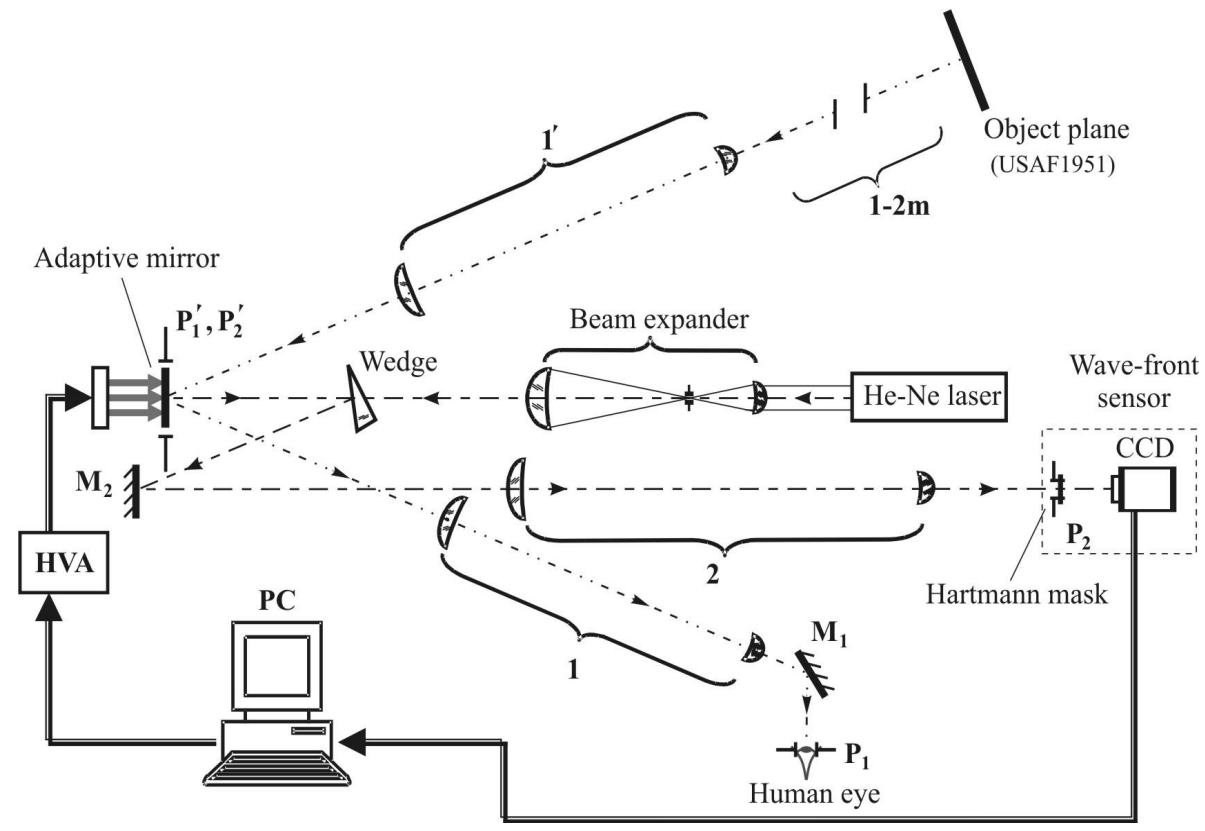

Figure 13. Experimental setup. The subjective loop is formed by the resolution target positioned in the object plane, the adaptive mirror and the human eye. System pupils are conjugated using telescopes. The objective loop consists of a laser, beam expander, deformable mirror and the Hartmann-Shack sensor with beam-forming optics. The 127-subaperture microlens array (Hartmann mask) is conjugated to the deformable mirror plane.

1. Subjective: the method improves the visual acuity of the test subject;

2. Objective: the correction figures obtained by the same individual using the deformable mirror in different correction cycles should be similar to each other.

3. The aberration coefficients, measured in different correction attempts, correlate to each other.

The experiments were conducted with two groups of individuals. The first group included 8 different individuals aged from 14 to 70 years. The second group included 12 Chinese students, with ages in the range 20 to 30 years. Each individual was instructed to improve their visual acuity as much as possible, by using the keyboard controls. Each person made 6 attempts to best adjust their vision, using 6 to 12 orthogonal modes. The subjective estimate of the visual acuity was obtained as the number of the contrast pattern clearly readable in the USAF test table. After each attempt, the figure of the deformable mirror was measured and Zernike coefficients and surface interferograms were recorded.

We did not stabilize the optical axis of the eye, using only a forehead and chin rest to stabilize the head position. All participants were instructed to keep the line of sight as stable as possible by looking always at the same area of the test pattern.

\section{EXPERIMENTAL RESULTS}

The results are summarized below:

- Most participants could reliably and repeatedly control their visual acuity by changing the amplitudes of up to 12 orthogonal correction modes, including coma, astigmatism, trefoil and spherical aberration.

- Participants with good eyes usually did not report any significant improvement of their visual acuity as a result of the optimization cycle over all low-order aberrations. 

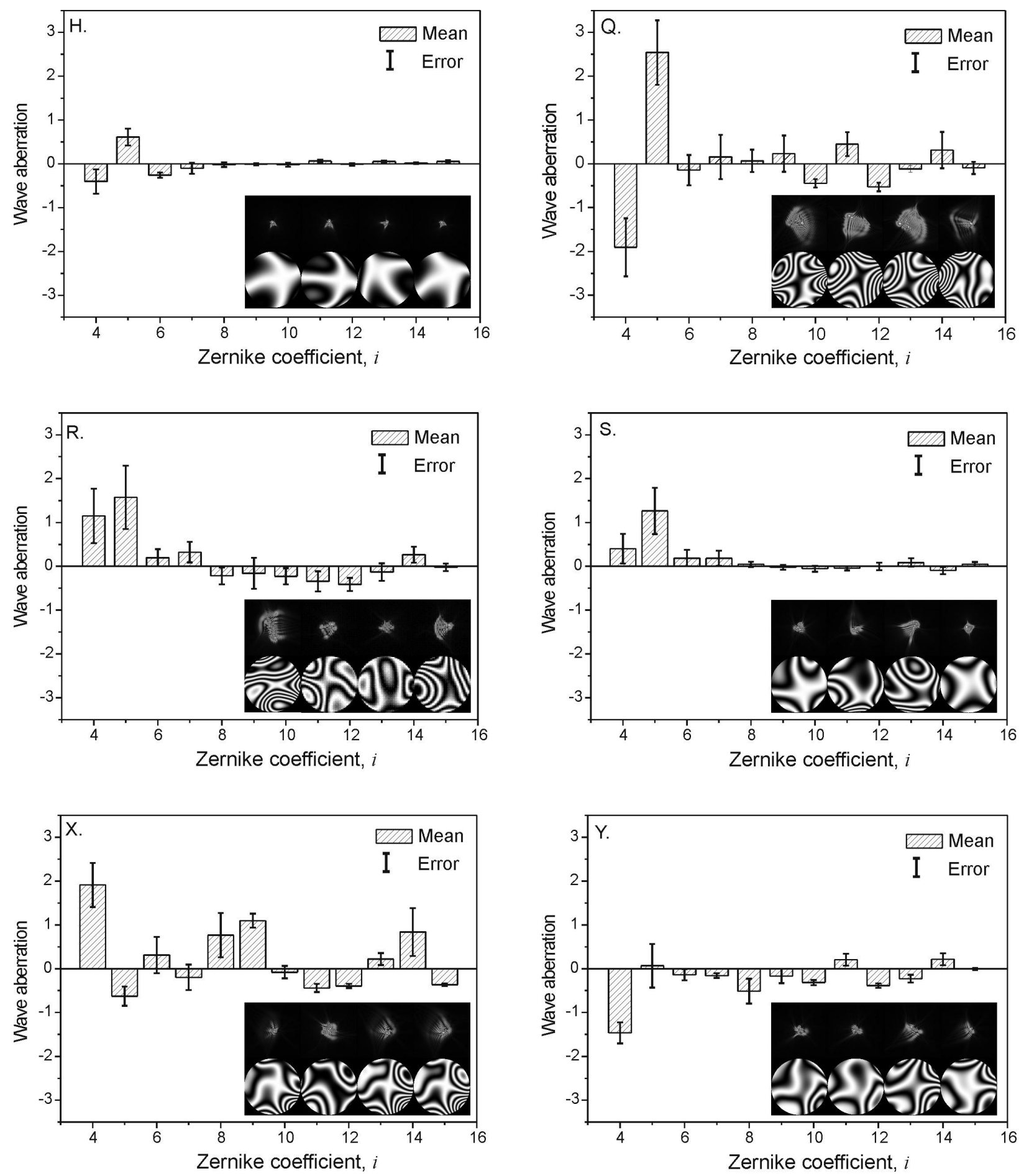

Figure 14. Experimental results obtained by 6 subjects, each subject making 6 correction attempts. In this graph we show the results of 4 most correlated attempts for each subject. 
- Some participants (presumably with stronger aberrations) reported moderate to significant improvement of their visual acuity, corresponding to as much as a doubling of the subjectively perceived resolving power.

- All participants described the feedback procedure as a rather tiring, resulting in tired eyes and a decreasing ability to use the system controls. After being introduced to the system, the participants usually achieved the best subjective result in the 3rd or 4th attempt; later attempts produced less impressive results due to eye tiredness.

- All participants reported that the correction quality was very sensitive to the movements of the eye.

Figure 14 shows the best correction results for 6 individuals from the second group of 12 students. The graph includes the values of Zernike terms, the corrector interferogram and the PSF, corresponding to the measured aberration.

In all cases the Zernike coefficients are grouped around the average values with moderate to good repeatability.

\section{CONCLUSIONS}

Finally, we designed and implemented an experimental setup, that allows for adjustment of up to 12 Zernike aberrations of the human eye, based on subjectively perceived sharpness of the observed test image. Using this setup we demonstrated that the major low-order aberrations of the human eye can be successfully corrected using subjective feedback based on perceived visual acuity.

\section{REFERENCES}

1. J. Liang, B. Grimm, S. Goelz, and J. F. Bille, "Objective measurement of wave aberrations of the human eye with the use of a hartmann-shack wave-front sensor," Journal of the Optical Society of America 11(7), pp. 1949-1957, 1994.

2. E. J. Fernandez, I. Iglesias, and P. Artal, "Closed-loop adaptive optics in the human eye," Optics Letters 26(10), pp. 746-748, 2001.

3. N. Doble, G. yoon, L. Chen, P. Bierden, S. Oliver, and D. R. Williams, "Use of a microelectromechanical mirror for adaptive optics in the human eye," Optics Letters 27(17), pp. 1537-1539, 2002.

4. H. Hofer, L. Chen, G.-Y. Yoon, B. Singer, Y. Yamauchi, and D. R. Williams, "Improvement in retinal image quality with dynamic correction of the eye's aberrations," Optics Express 8, pp. 631-643, 2001.

5. S. Bara, T. Mancebo, and E. Moreno-Barriuso, "Position tolerances for phase plates compensating aberrations of the human eye," Applied Optics 39(19), pp. 3413-3420, 2000.

6. R. Navarro, E. Moreno-Barriuso, S. Bara, and T. Mancebo, "Phase plates for wave-aberration compensation in the human eye," Optics Letters 25(4), pp. 236-238, 2000.

7. G. V. M. L. A. Naumov, "On the possibility of intraocular adaptive optics," Opt. Express 11, pp. 810-817, 2003.

8. "http://okotech.com."

9. C. Paterson and I. M. C. Dainty, "A low cost adaptive optics system using a membrane mirror," Optics Express 6, pp. 175-185, 2000. 